\title{
VALORACIÓN DEL CAPITAL HUMANO EN EL DESARROLLO DEL ENCARGO DE AUDITORIA: UN ANÁLISIS DESDE LAS NORMAS \\ INTERNACIONALES DE CONTROL DE CALIDAD
}

\section{ASSESSMENT OF HUMAN CAPITAL IN THE DEVELOPMENT OF THE AUDIT ORDER: AN ANALYSIS FROM THE INTERNATIONAL STANDARDS OF QUALITY CONTROL}

\section{Recebimento: 16/05/2017- Aceite: 12/11/2017- Publicação: 23/12/2017 \\ Processo de Avaliação: Double Blind Review}

\author{
Ludivia Hernandez Aros ${ }^{1}$ \\ Profesora Investigadora Facultad de Contaduría Pública Universidad Cooperativa de \\ Colombia, Sede Ibagué, Colombia. \\ Estudiante de Maestría en Auditoría y Gestión Empresarial de la Universidad UNINI - \\ Puerto Rico \\ Especialista en Revisoría Fiscal y Control de Gestión de la Universidad Cooperativa de \\ Colombia ludivia.hernandez@campusucc.edu.co
}

Fernando Gutiérrez Pórtela

Profesor Universidad Cooperativa de Colombia Sede Ibagué.

Magister en Software Libre, Ingeniero de Sistemas.

fernando.gutierrez@campusucc.edu.co

Luis Eduardo Parra Hernández

Estudiante de pregrado de la Universidad Cooperativa de Colombia.

luis.parrah@campusucc.edu.co

Martha Lucia Mayolo Bonilla

Estudiante de pregrado de la Universidad Cooperativa de Colombia - sede Ibagué, del programa de Contaduría Pública.

martha.mayolob@campusucc.edu.co

\section{RESUMEN}

Con el desarrollo del artículo se pretende valorar el capital humano en el desarrollo del encargo de auditoria desde las normas internacionales de control de calidad, para lo cual se tuvo en cuenta la siguiente metodología, principalmente se indago sobre las teorías del capital intelectual y sus componentes, posteriormente se profundizo sobre el capital humano, para después establecer la relación del capital humano con las Normas Internacionales de Control de Calidad-NICC. Se obtuvo que la valoración del capital humano en el desarrollo de una auditoria, se obtiene a partir de una serie de indicadores, los cuales relacionan cualidades de los empleados con la situación de un ente, cabe anotar que dicha valoración es de carácter subjetivo, pero ayuda a tener conocimiento sobre el nivel del capital humano con el cual se cuenta a la hora de realizar un encargo.

\footnotetext{
${ }^{1}$ Autor para correspondência: Universidad Cooperativa de Colombia - Sede Ibagué. Edificio Urrutia (Calle $10 \mathrm{~N}^{\circ}$ 1-120 Bloque $\mathrm{N}^{\circ}$ 2). Ibagué ( Tol). Colombia
}

Revista ENIAC Pesquisa, Guarulhos (SP), V.6, n.2, jun.- dez. 2017 
PALABRAS CLAVE: Capital humano, valoración del capital humano, auditoria, NICC

\section{ABSTRACT}

With the development of the article, the aim is to assess the human capital in the development of the audit commission from the international standards of quality control, for which the following methodology was taken into account, mainly we inquired about the theories of intellectual capital and its components, later on, we deepened on the human capital, to later establish the relationship of the human capital with the International Norms of Control of Quality-NICC-. It was obtained that the valuation of the human capital in the development of an audit, is obtained from a series of indicators, which relate the qualities of the employees with the situation of an entity, it should be noted that said evaluation is of a subjective nature, but It helps to have knowledge about the level of human capital that is counted at the time of making an order.

KEYWORDS: Human capital, valuation of human capital, audit, NICC

\section{INTRODUCCIÓN}

Considerando lo anterior, el propósito del artículo es valorar el capital humano en el desarrollo del encargo de auditoria desde las normas internacionales de control de calidad, para lo cual se desarrollan los siguientes objetivos.

En primer lugar, es necesario identificar por medio de un barrido teórico los elementos y variables para valorar el capital humano en la aplicación de las normas internacionales de control de calidad, con el fin de tener las bases apropiadas para desarrollar el tema objeto de estudio.

Una vez teniendo claro los conceptos se pretende caracterizar los elementos y variables del capital humano a tener en cuenta en la aplicación de las normas internacionales de control de calidad para un encargo, por medio de indicadores y haciendo uso de un esquema que permita tener claros los elementos y variables anteriormente estudiadas.

Para finalizar se va a diseñar un Prototipo de modelo de tablero de control que genere indicadores de medición del capital humano para las normas internacionales de control de calidad, para de esta manera poder tener un método de valorizar el capital humano en el desarrollo de un encargo.

El documento se estructura en tres secciones, la primera sección se enfoca en entender el concepto de capital intelectual, capital humano y su relación con las normas internacionales de control de calidad, en la segunda sección se encarga de aclarar a fondo el capital humano, sus características, elementos y la forma de medir el mismo dentro de una organización, y por último se determina el objeto de estudio a partir de la elaboración de un tablero de control que perita valorar el capital humano dentro de las organizaciones.

\section{METODOLOGÍA}

El presente estudio es un estudio de carácter descriptivo, documental en que la información es recolectada a través de un barrido literario y a partir de lo anterior se tuvo en 
cuenta las siguientes fases. Inicialmente, se indago en las fuentes de información los conceptos de capital intelectual, capital humano, y los elementos que lo componen; seguidamente se analizó las formas de medir el capital humano dentro de las organizaciones; con la tabulación de indicadores necesarios para medir el capital humano en una organización, donde se caracterizó sus las variables y por último, se diseñó un tablero de control para valorizar las variables del capital humano dentro de una organización.

\section{MARCO TEÓRICO}

Ante la importancia que en la actualidad tiene para las organizaciones el capital intelectual o más conocido como activo intangible, algunos autores lo definen como "El capital intelectual hace referencia al conjunto de activos intangibles que poseen las empresas, y que conjuntamente con los activos tangibles, forman parte de su patrimonio" (Alama, Navas y de Castro (2008, p.56); es decir que hace parte de todas las organizaciones, y su buen manejo ayuda al cumplimiento de estrategias que se enfocan en el logro del éxito empresarial.

En la definición es preciso aclarar, que/ los activos intangibles no solo son los conocidos contablemente (Marcas, know how, licencias, etc.), si no también, encierra los factores internos que son propios de una empresa. Para enfocar el concepto, Edvinsson y Malone (Como se citó en Medina, A. J., Gonzales, A. y Pérez, E., 2008) explican que la organización no solo se debe preocupar por resultados financieros, sino también aquellos "valores ocultos", intangibles que afectan a la organización en el largo plazo.

La anterior explicación complementa la definición inicial del capital intelectual, ya que se enfoca como un valor intangible y de primera necesidad, siendo el factor con más relevancia dentro de las organizaciones por su extensión, por que abarca todos los componentes de una empresa. Dentro de las definiciones de capital intelectual, se expone que se compone de diferentes clases; al respecto, Medina et al., (2007) argumenta que "En los últimos años parece existir un cierto consenso en, (...).dividir el capital intelectual en tres componentes: el capital humano, el capital estructural y el capital relacional" (p. 102).

En la figura 1 se evidencia la configuración del capital intelectual. Como se refleja en la figura 1, el capital humano se define según López y Grandino (2005) como el conocimiento tácito o explícito, poseído por las personas y equipos, el cual se complementa con la capacidad de regenerar el conocimiento, es decir, con la capacidad de aprender y su efecto diferenciador reside en que el capital humano no es propiedad de la empresa. 


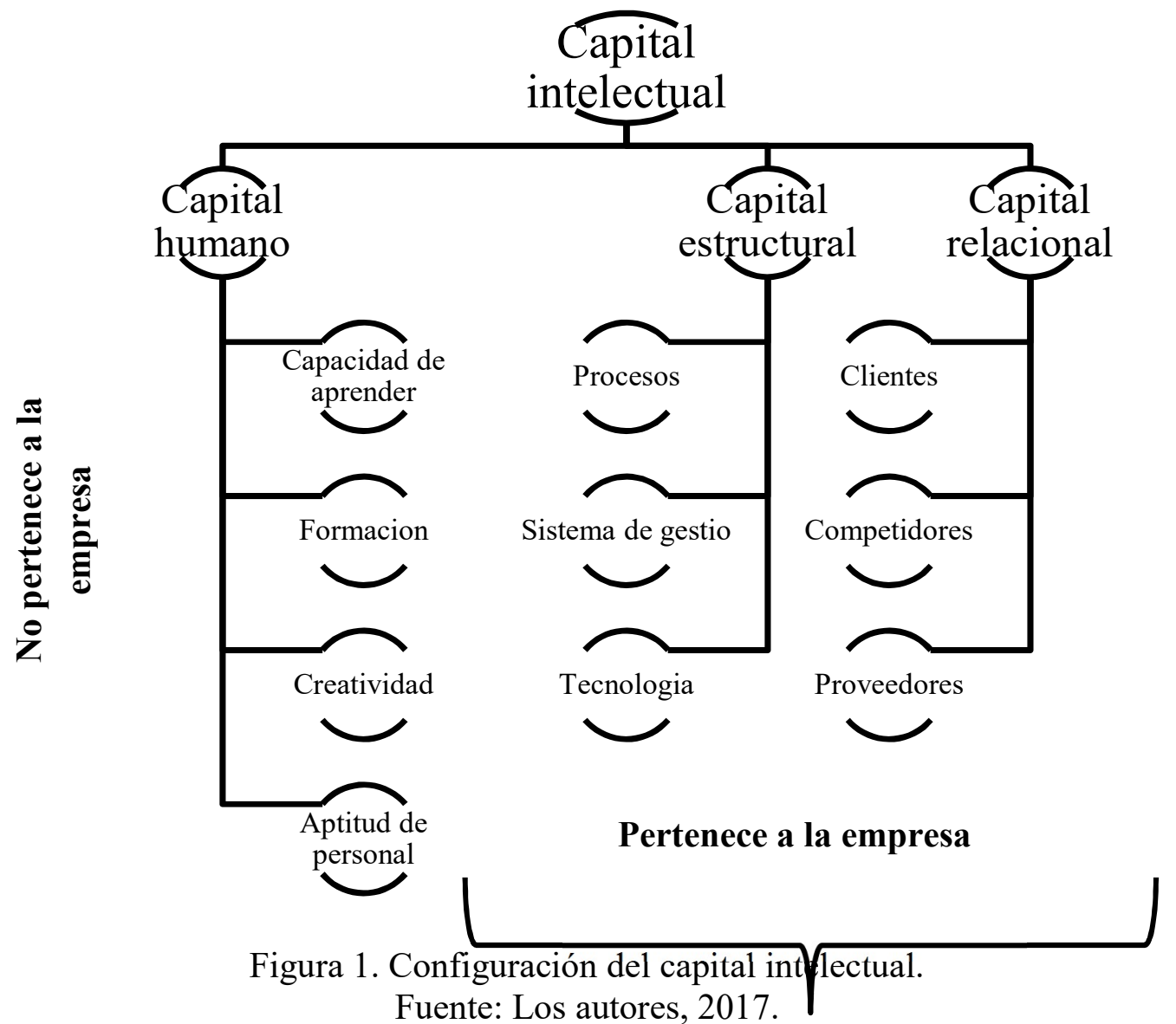

La anterior definición hace referencia a las destrezas y conocimientos de los trabajadores de la compañía, y la buena forma en que estos sean utilizados con el fin de encontrar el desarrollo de la empresa, y su sostenibilidad.

El capital estructural, lo definen varios autores como Brooking, 1996; Bontis, 1998; Bueno, 1998; Wang \& Chang, 2005; Jardon, C. M., Gutawski, R. S., Martos, M. S., Aguilar, C. A. y Barajas, A., 2007 (Como se citó en Martos, M. S., Jardon, C. M. y Florián, P., 2008) como aquel que:

\footnotetext{
Suele estar integrado por aspectos referidos a la organización y a la tecnología. Entre los primeros se incluye la cultura organizacional, esto es, el conjunto de valores que impulsados por la directiva sirven como modelos de comportamiento en la organización. También se incluye la forma de organizar los distintos trabajos que realiza la empresa y la alineación del personal con la estrategia, que facilita la conexión de los trabajadores con la dirección. Entre los aspectos tecnológicos, se habla de la tecnología introducida en cada uno de los productos/servicios o procesos que realiza la empresa, y muy especialmente se debe tener en cuenta la capacidad de información tecnológica, que permite la captación de tendencias y avances en ciencia y tecnología (p.73).
}

En complemento a la definición anterior López, y Grandino (2005) argumentan que "El capital estructural pertenece a la empresa e incluye los protocolos de transmisión de información formales. Un sólido capital estructural facilita los flujos de información, incidiendo positivamente sobre la eficiencia de la organización" (p. 52). En síntesis el capital estructural es la capacidad organizativa, tecnológica y comunicativa de cada organización para transmitir el correcto funcionamiento de la empresa. 
El tercer y último elemento del capital intelectual, se refiere a todas las relaciones del ente económico con su exterior. López y Grandino (2005), sostienen que el capital relacional "Engloba el valor del conjunto de relaciones que una empresa mantiene con el exterior. Dichas relaciones se consideran en un bloque distinto por la importancia y características diferenciadas que poseen" (p. 52).

En ampliación al capital relacional Martos et al. (2008) sostiene que "El capital relacional (CR) incluye las relaciones con el entorno, y más específicamente con los agentes económicos que participan en las diferentes fases de la cadena de valor del producto: los proveedores, los competidores y los clientes." (p. 74). Por lo que se puede inferir que el capital relacional, es de propiedad de la empresa y su medición puede ser difícil de realizar, debido a que las relaciones comerciales de una organización son complicadas de valorar dependiendo del tipo de ente.

Es claro que los elementos anteriormente descritos se encuentran presentes en todas las organizaciones, algunos elementos con más presencia que otros, eso depende de cada tipo de organización; pero sin lugar a duda el capital humano es el elemento que no debe faltar dentro de las empresas, pues no es posible concebir una empresa sin la presencia del capital humano, según sostienen López y Grandino (2005) "El capital humano es, en la mayor parte de las organizaciones, el activo más importante, ya que genera el capital estructural y relacional de la compañía" (p. 59). Es decir a partir del capital humano se genera el capital relacional y el capital estructural en una compañía, por ello se hace importante profundizar a cerca del capital humano, según (Contreras, 2012) se refiere al capital humano así,

El capital humano constituye actualmente un factor que agrega valor a las organizaciones, este valor se hace aún más relevante cuando el conocimiento se coloca en función del logro de los objetivos de la organización. El capital humano depende en gran medida de la capacidad que tengan las organizaciones para aprovechar y desarrollar el conocimiento. El proceso idóneo para perfeccionar los conocimientos y desarrollar el capital humano es justamente el de capacitación y desarrollo de personal (p. 14).

Davenport (Como se citó en Hernández, F. 2006) define los elementos del capital humano:

Capacidad, comportamiento y esfuerzo y señala que; la capacidad se divide en tres subcomponentes, conocimiento, habilidad y talento; el conocimiento lo representa como el contexto intelectual dentro del cual interactúa una persona; la habilidad como la familiaridad con los medios y métodos para realizar una determinada tarea. Abarca desde las habilidades físicas hasta un aprendizaje especializado; el talento como la facultad innata para realizar una tarea específica sinónimo de actitud.

El comportamiento es la forma de manifestar la conducta de los valores éticos, creencias y relaciones ante el mundo, el comportamiento combina respuestas inherentes adquiridas con situaciones y estímulos situacionales; el esfuerzo es la aplicación consciente de los recursos mentales y físicos a un fin concreto, promueve tanto la habilidad como el conocimiento y el talento.

Los elementos anteriormente mencionados, se estructuran y relacionan como lo refleja a continuación la figura 2 .

Revista ENIAC Pesquisa, Guarulhos (SP), V.6, n.2, jun.- dez. 2017 


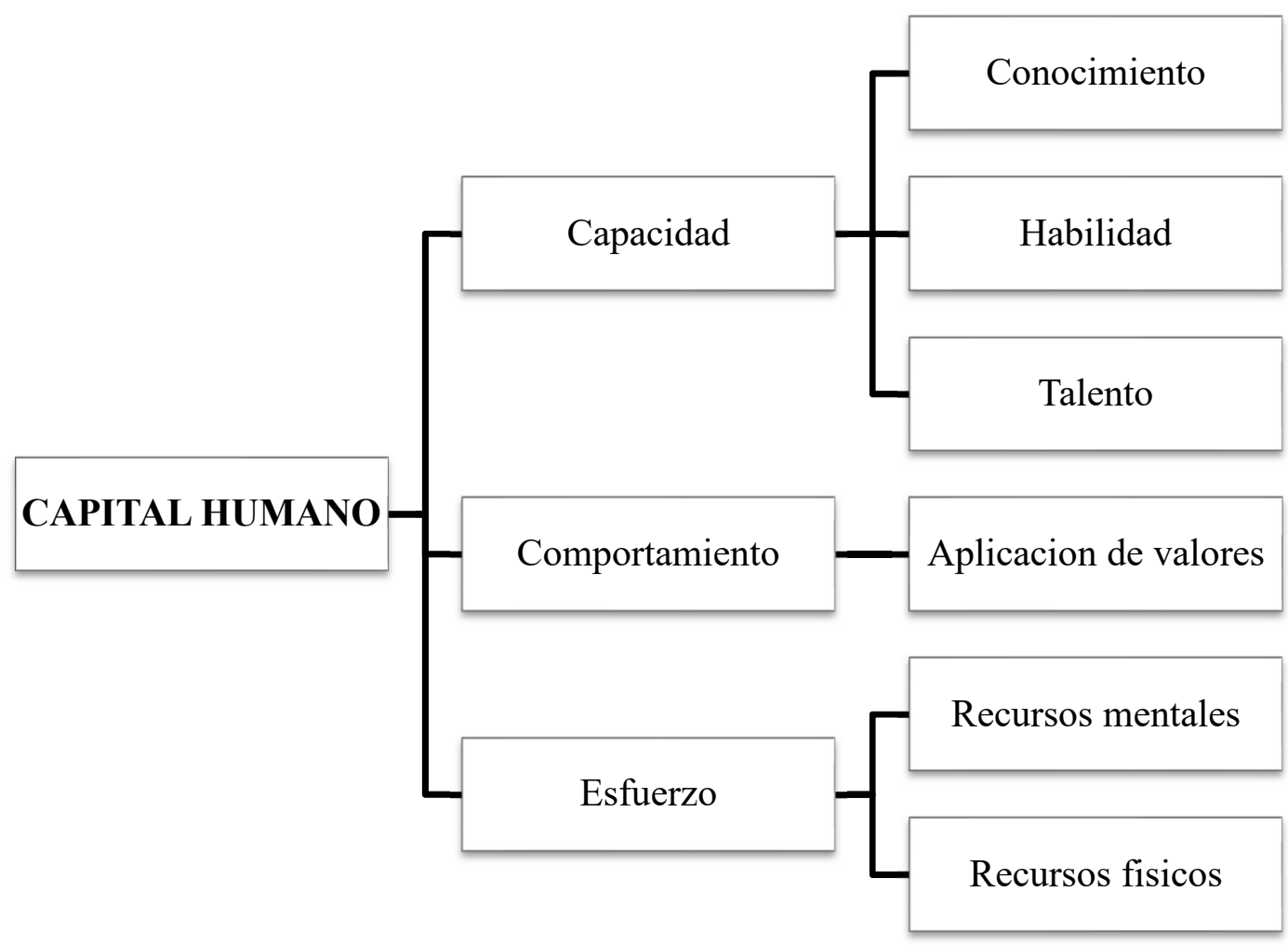

Figura 2: Elementos del capital humano.

Fuente: Los autores, 2017.

Una vez entendidos los elementos que componen el capital humano, es necesario buscar la manera de medir el capital humano dentro de las organizaciones, para lo cual López y Grandino (2005) argumentan que:

Muestra que los indicadores de capital humano se pueden agrupar en seis categorías básicas: 1) Perfil del empleado, 2) Rotación del personal, 3) Educación, 4) Compromiso y motivación, 5) Formación, y 6) Resultados.

En la sección del perfil del empleado se proporciona la información sobre la distribución de edades y sexo de los empleados, número de empleados que trabajan en los departamentos de producción, distribución, tecnología de la información, ventas, marketing y administración. En cuanto a la rotación del personal incluye diversos datos sobre los empleados contratados, aquellos que dejan la empresa, porcentaje de rotación de personal, etc.

En el aspecto de educación muestra información sobre la formación académica de los empleados y su experiencia (no cualificados, cualificados, doctores, con experiencia internacional, etc.). Entre los indicadores de compromiso, se incluyen los siguientes: porcentaje de personal ascendido/total del personal, antigüedad de los empleados, etc. Entre los indicadores de motivación se encuentran el porcentaje de los empleados que sienten un reconocimiento explícito en la empresa, \% de empleados que consideran que sus opiniones son tenidas en consideración o que están satisfechos con su entorno de trabajo, etc.

La formación incluye indicadores sobre las inversiones en formación para empleados que realiza la empresa. Entre ellos destacan los siguientes: Número de días de formación por 
empleados, ratio de horas de formación/horas de trabajo (anual), inversión en formación por empleado al año, etc.

Tabla 1 Indicadores del capital humano.

\begin{tabular}{|c|c|c|}
\hline Categoría & Indicadores & Interpretación \\
\hline \multirow{3}{*}{$\begin{array}{l}\text { Perfil del } \\
\text { empleado. }\end{array}$} & a-Número de empleados según el sexo & Distribuye los empleados según el sexo \\
\hline & $\begin{array}{l}\text { b- Distribución de empleados según la } \\
\text { edad. }\end{array}$ & Determina la edad promedio de la empresa \\
\hline & c-Distribución de empleados por áreas. & $\begin{array}{l}\text { Distribuye loe empleados según las áreas de la } \\
\text { empresa. }\end{array}$ \\
\hline \multirow{4}{*}{$\begin{array}{l}\text { Rotación del } \\
\text { personal. }\end{array}$} & a-Número de empleados nuevos. & Nuevas contrataciones en el periodo. \\
\hline & b- Número de empleados retirados. & Empleados que se retiran por decisión propia \\
\hline & c-Número de empleados despedidos. & $\begin{array}{l}\text { Empleados que son retirados por decisión de la } \\
\text { empresa. }\end{array}$ \\
\hline & $\begin{array}{l}\text { d- Número de ingresos/número de } \\
\text { salidas* } 100 .\end{array}$ & Porcentaje de reemplazo del personal \\
\hline \multirow{2}{*}{ Educación. } & $\begin{array}{l}\text { a- Distribución de empleados según } \\
\text { nivel educativo. }\end{array}$ & $\begin{array}{l}\text { Determina qué nivel educativo tiene los } \\
\text { empleados. }\end{array}$ \\
\hline & b- Años de experiencia por empleado. & $\begin{array}{l}\text { Determina cuanta experiencia tiene la empresa } \\
\text { en sus empleados. }\end{array}$ \\
\hline \multirow{5}{*}{$\begin{array}{l}\text { Compromiso } \\
\text { y motivación. }\end{array}$} & $\begin{array}{l}\text { a-Número de empleados } \\
\text { ascendidos/empleados totales. }\end{array}$ & $\begin{array}{l}\text { Determina el porcentaje de ascensos de los } \\
\text { empleados }\end{array}$ \\
\hline & b- Antigüedad de los empleados. & $\begin{array}{l}\text { Determina la experiencia de los empleados en } \\
\text { la empresa, es de gran utilidad para evitar } \\
\text { reiteración de errores. }\end{array}$ \\
\hline & $\begin{array}{l}\text { c- } \% \text { de empleados que se sienten con } \\
\text { reconocimiento. }\end{array}$ & $\begin{array}{l}\text { Define el porcentaje de empleados que se } \\
\text { sienten reconocidos por su labor }\end{array}$ \\
\hline & $\begin{array}{l}\text { d- } \% \text { de empleados que sienten que sus } \\
\text { opiniones son tenidas en cuenta. }\end{array}$ & $\begin{array}{l}\text { Señala el porcentaje de empleados que se } \\
\text { sienten parte de la empresa. }\end{array}$ \\
\hline & $\begin{array}{l}\text { e- } \% \text { de empleados que se sienten } \\
\text { satisfechos con su labor. }\end{array}$ & $\begin{array}{l}\text { Define los empleados que spn felices } \\
\text { realizando su labor. }\end{array}$ \\
\hline \multirow{4}{*}{ Formación. } & $\begin{array}{l}\text { a-Inversión en formación/empleados } \\
\text { totales. }\end{array}$ & $\begin{array}{l}\text { Mide la inversión que se realiza por empleado } \\
\text { para generar bienestar en los mismos. }\end{array}$ \\
\hline & $\begin{array}{l}\text { b- Número de días en formación por } \\
\text { empleado. }\end{array}$ & $\begin{array}{l}\text { Define el número de días que los empleados } \\
\text { invierten en formación. }\end{array}$ \\
\hline & $\begin{array}{l}\text { c- Aplicación de pruebas para } \\
\text { comprobar los procesos internos y su } \\
\text { eficiencia. }\end{array}$ & $\begin{array}{l}\text { Mide el grado de cumplimiento y } \\
\text { conocimiento de los procesos internos de la } \\
\text { compañía. }\end{array}$ \\
\hline & $\begin{array}{l}\text { d- Numero de formaciones realizadas en } \\
\text { el año. }\end{array}$ & $\begin{array}{l}\text { Establece el número de formaciones que se } \\
\text { realizan en un periodo }\end{array}$ \\
\hline Resultados. & $\begin{array}{l}\text { a- } \% \text { de empleados que se sienten } \\
\text { satisfechos en la empresa. }\end{array}$ & $\begin{array}{l}\text { Define el porcentaje de los empleados que se } \\
\text { encuentran satisfechas en la compañía }\end{array}$ \\
\hline
\end{tabular}

Fuente: Los autores, 2017. 
Por ultimo está el resultado, en el cual este grupo de indicadores muestra la satisfacción global con el trabajo desempeñado. Generalmente se mide a través de un índice de satisfacción del empleado.

A partir de la información anterior, se puede construir la tabla 1, que resume lo indicadores de capital humano por categoría.

En conclusión, el capital humano se puede catalogar como el factor de mayor relevancia dentro de la definición del capital intelectual y dentro de las organizaciones, ya que a partir del mismo se genera valor y desarrollo en las empresas. Su medición debe ser constante, porque no existe una empresa sin capital humano y este debe mantenerse en un nivel que sea beneficioso para la compañía y produzca bienestar para los empleados.

Es preciso aclarar que el capital humano en caso contrario a los bienes materiales que se deprecian, se puede valorizar, puesto que el ser humano está en constante aprendizaje $\mathrm{y}$ al ser una persona pensante puede contribuir a la empresa en su desarrollo económico.

En la actualidad, y con el proceso de globalización se ha implementado un modelo de información que propenda por estandarizar la información financiera de las compañías, un método para cumplir esta estandarización son las Normas Internacionales de Control de Calidad (NICC), y es allí donde el capital humano juega un papel importante, pues a partir de un capital humano competente se garantiza la aplicación de las NICC, y por consiguiente se obtiene una información veraz y fiel.

Ahora bien, en el marco de las Normas Internacionales de Control de Calidad (NICC) mediante el decreto 2420 del 14 de diciembre de 2015, se refiere a las NICC en el artículo 1.2.1.1. Como elemento dentro del marco normativo de las Normas de Aseguramiento de la Información (NAI). Y la aplicación de las NICC, según el artículo 1.2.1.7. Será a los Contadores Públicos que presten servicios revisoría fiscal, auditoría información financiera, revisión de información financiera histórica u otros trabajos de aseguramiento, aplicarán en sus actuaciones profesionales las NICC.

Dicho de otro modo, las NICC son parte de las NAI; y su aplicación se enmarca a las actividades de aseguramiento de la información, aplicadas por los contadores públicos y/o empresas dedicadas a las profesiones de la contabilidad, con la finalidad de garantizar que los resultados de cualquier auditoria aplicada sean ajustados a la realidad.

Es entonces donde se hace necesario darle una valoración al capital humano, desde la perspectiva de las NICC, pues estas exigen calidad dentro de los trabajos de auditoria, y dicha calidad es resultado tangible de los procesos aplicados por un capital humano calificado y de alto valor.

Dentro del decreto 2420 del 24 de diciembre de 2015, se establecen el objetivo de la firma de auditoría es el establecimiento y el mantenimiento de un sistema de control de calidad que le proporcione una seguridad razonable de que la firma de auditoría y su personal cumplen las normas profesionales y los requerimientos legales y reglamentarios aplicables; y que además los informes emitidos por la firma de auditoría o por los socios de los encargos son adecuados en función de las circunstancias.

En las NICC, la firma de auditoría establecerá y mantendrá un sistema de control de calidad que comprenda políticas y procedimientos que contemplen cada uno de los

Revista ENIAC Pesquisa, Guarulhos (SP), V.6, n.2, jun.- dez. 2017 
siguientes elementos: responsabilidades de liderazgo en la calidad dentro de la firma de auditoría; requerimientos de ética aplicables; aceptación y continuidad de las relaciones con clientes y de encargos específicos; recursos humanos; realización de los encargos; seguimiento.

Finalmente, la firma de auditoría establecerá políticas y procedimientos diseñados para promover una cultura interna en la que se reconozca que la calidad en la realización de un encargo es esencial. Dichas políticas y procedimientos, requerirán que el presidente ejecutivo (o equivalente) de la firma de auditoría o, cuando proceda, el consejo directivo de socios (o equivalente) de la firma de auditoría, asuman la responsabilidad última del sistema de control de calidad de la firma de auditoría.

\section{RESULTADOS}

En la aplicación de las normas internacionales de control de calidad es necesario hacer una revisión al capital humano, el cual es un elemento muy importante a lo largo de largo de todo el proceso de aplicación. Tan solo el hecho de implementar un sistema que garantice la calidad de una auditoria, conlleva a tener un capital humano idóneo, capaz, apto, calificado y cualificado, de tal modo que este sea la base fundamental a partir de la cual la calidad en una auditoria se va a garantizar.

Con la anterior aclaración, se entiende que en un sistema de control de calidad para el desarrollo de una auditoría, el capital humano desempeña un rol importante; por lo anterior en la tabla siguiente, se describen los elementos y variables a tener en cuenta en la aplicación de las normas internacionales de control de calidad.

\begin{tabular}{|c|c|c|c|}
\hline \multicolumn{4}{|c|}{ Elementos y variables del capital humano } \\
\hline Variables & Elementos & Riesgos & $\begin{array}{l}\text { Nivel de } \\
\text { riesgo }\end{array}$ \\
\hline \multirow{7}{*}{ 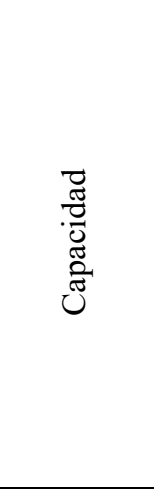 } & \multirow{4}{*}{ Conocimiento } & Falta de conocimiento sobre el desarrollo de un encargo & Alto \\
\hline & & Educación limitada para el desarrollo de encargos. & Alto \\
\hline & & $\begin{array}{l}\text { Caracterización de perfiles inadecuada para el desarrollo de un } \\
\text { encargo }\end{array}$ & Alto \\
\hline & & $\begin{array}{l}\text { Conocimientos limitados sobre el desarrollo de un encargo de } \\
\text { auditoria. }\end{array}$ & Alto \\
\hline & \multirow[t]{2}{*}{ Habilidad } & $\begin{array}{l}\text { Constante cambios en el personal, lo que genera reprocesos de } \\
\text { capacitación }\end{array}$ & Bajo \\
\hline & & Falta de habilidades y técnicas en la aplicación de auditorías. & Alto \\
\hline & Talento & $\begin{array}{l}\text { Falta de actualizaciones para aprender nuevas técnicas en el } \\
\text { desarrollo de un encargo. }\end{array}$ & Medio \\
\hline \multirow{2}{*}{ 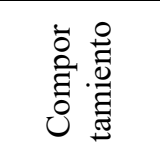 } & $\begin{array}{l}\text { Aplicación de } \\
\text { valores }\end{array}$ & $\begin{array}{l}\text { Carencia de valores y ética en el desarrollo de encargos de } \\
\text { auditoria. }\end{array}$ & Alto \\
\hline & Motivación & Déficit de motivación y compromiso para desarrollar el trabajo. & Medio \\
\hline \multirow{3}{*}{ 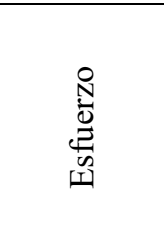 } & $\begin{array}{l}\text { Recursos } \\
\text { físicos }\end{array}$ & $\begin{array}{l}\text { Escasez de recursos físicos en el personal que desarrolla el } \\
\text { encargo. }\end{array}$ & Bajo \\
\hline & $\begin{array}{l}\text { Recursos } \\
\text { mentales }\end{array}$ & $\begin{array}{l}\text { Déficit de capacidad mental en el desarrollo del encargo de una } \\
\text { auditoria. }\end{array}$ & Alto \\
\hline & Resultado & Insatisfacción con el resultado del trabajo desempeñado. & Medio \\
\hline
\end{tabular}

Tabla 2: Riesgos del capital humano en la aplicación de las NICC, 2017.

Fuente: Los autores, 2017. 
En la matriz anterior se establece la relación entre las variables del capital humano y los riesgos que presenta cada una de ellas al momento de aplicar las NICC. Se divide en tres grande grupos, capacidad, comportamiento y esfuerzo. La capacidad de manera general encierra todas las aptitudes que requiere una persona, por otro lado, el comportamiento y el esfuerzo son de índole personal por lo que agrupan las actitudes del capital humano.

En cuanto a la capacidades se encuentran divididas en tres clases, tales como conocimiento, habilidad y talento, las cuales se complementan entre sí; en el conocimiento es donde se encuentran los riesgos más altos, todo debido a que las NICC requieren de personal con conocimiento suficiente en el desarrollo del encargo ; en pocas palabras, en el desarrollo de un sistema de control de calidad, un personal sin el conocimiento adecuado tiene alta probabilidad de fallar y desvirtuaría el objetivo de las NICC.

La habilidad son todas las técnicas que desarrolla el personal durante su labor, esta puede adquirirse con el tiempo y por tanto se puede concebir como la experiencia del personal a lo largo de su vida. El riesgo de la habilidad radica en dos puntos claves, el primero es el constante cambio de personal, pues si se realiza de forma frecuente desencadenaría en reprocesos de inducción, lo cual no estaría garantizando la continuidad del personal y las habilidades no podrían desarrollarse de forma correcta. El segundo punto clave es la falta de habilidad y técnica que pueda tener una persona en el desarrollo de una auditoria, esta carencia de habilidades da paso a ser ineficiente en su labor.

El talento, es la capacidad de una persona para adquirir nuevos conocimientos y técnicas, por el contrario de la habilidad, el talento no se adquiere con experiencia, es decir el talento es innato en cada persona, pero si es complemento de las habilidades, pues si una persona es talentosa, tiene altas probabilidades de adquirir fácilmente las habilidades. El riesgo que se expone en el talento es la falta de actualizaciones al personal, debido a que si no hay constante preparación al personal, se estaría desaprovechando el talento del capital humano. Esta trilogía de capacidades se complementan entre si pues el talento es algo que se alimenta con el conocimiento, y proporciona el desarrollo de las habilidades.

El comportamiento es considerado como una actitud, y hace referencia a los valores y la motivación del personal; por un lado los valores son subjetivos y están inmersos en el personal, por lo cual en ocasiones se hace difícil medirlo y quizás la única forma de lograr tener concepto de los valores de una persona es a través del desarrollo de relaciones directas con el personal, aunque el concepto que se obtenga sobre el comportamiento será muy subjetivo y varía de acuerdo a cada persona. Una vez se ha obtenido un concepto sobre los valores del personal, se puede determinar si estos valores son adecuados y suficientes para desarrollar un encargo de auditoria y es allí donde se refleja el riesgo de que los valores inmersos en cada persona sean los adecuados.

La motivación al personal es objetiva y recae en gran proporción en la dirección del encargo, de ella depende que el personal este en constante motivación y dispuesta a desarrollar los objetivos de un encargo, el riesgo radica cuando no existe motivación para desarrollar los objetivos planteados y la dirección no logra generar el compromiso de su capital humano con el desarrollo del trabajo.

En la última variable se encuentra el esfuerzo, y es considerado al igual que el comportamiento como una actitud del personal, claro está que la dirección juega un papel relevante en relación con el esfuerzo, debido a que una parte del esfuerzo recae en la dirección y su compromiso para proveer los recursos necesarios que permitan alcanzar los 
resultados. El esfuerzo se compone por los recursos físicos, los recursos mentales y los resultados. Los recursos físicos son donde se responsabiliza la dirección, ya que se compone por todas las herramientas tangibles que suministre la dirección para desarrollar el encargo, su riesgo se basa en que los recursos sean insuficientes y la falta de los mismos compromete la ejecución del encargo.

En cuanto a los recursos mentales, pertenecen al personal y es considerado como las capacidades para implementar de forma correcta los recursos físicos, y existe riesgo cuando los recursos mentales del persona no son los adecuados para aplicar los recursos físicos, es correcto afirmar que los recursos físicos y los recursos mentales son complementarios, pues deben existir los dos tipos de recursos para cumplir los objetivos planteados; por ultimo están los resultados, allí se reflejan la aplicación de todo el esfuerzo y todo el trabajo desarrollado, su riesgo es que el personal quede insatisfecho con el resultado obtenido, pues sentirá frustración en todo lo que ha realizado.

En cuanto a los riesgos planteados, existe una serie de posibles soluciones encaminadas a reducir los riesgos, estas se gestan a partir de la selección adecuada de personal, garantizando que el capital humano seleccionado sea el más idóneo para el desarrollo de las NICC. La dirección también tiene incidencia en la reducción de los riesgos, pues debe conservar un capital humano satisfecho, comprometido y actualizado, para lo cual debe destinar recursos con el fin de generar las características planteadas.

Ahora bien, un tablero de control que genere indicadores de medición del capital humano para las normas internacionales de control de calidad es significativamente importante para el auditor en su encargo, ya que se convierte en una herramienta importante con indicadores subjetivos y cuantificables que a través de los mismos muestran el grado de cumplimiento del capital humano para el desarrollo del encargo.

Es así como el perfil del empleado y su formación, hacen parte de valoraciones subjetivas, de gran peso en un sistema de control de calidad para el desarrollo de una auditoría; la rotación del personal, el compromiso y motivación, y los resultados, hacen parte de los indicadores cuantificables.

A continuación, en la siguiente tabla se muestra en detalle un prototipo de tablero de control, para la medición del capital humano para el desarrollo del encargo. 


\section{TABLERO DE CONTROL PARA MEDICIÓN DEL CAPITAL HUMANO}

\begin{tabular}{|c|c|c|c|c|c|c|c|}
\hline $\begin{array}{c}\text { Tipo de } \\
\text { indicador }\end{array}$ & Categoría & Indicador & Descripción-Formula & Resultado & Ppto. & Diferencia & $\begin{array}{c}\% \\
\text { cumplimiento }\end{array}$ \\
\hline \multirow{11}{*}{ 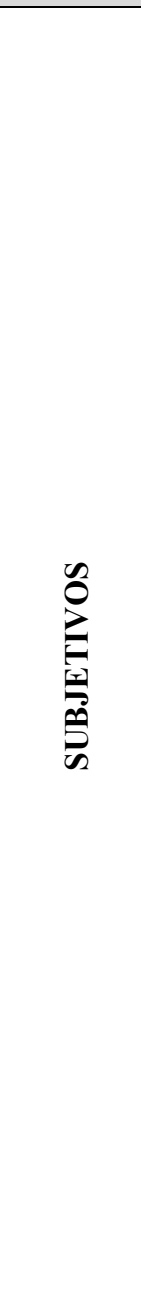 } & \multicolumn{3}{|c|}{ 1. Perfil del empleado. } & & & & \\
\hline & & Distribución de empleados & $\begin{array}{l}\text { Número de empleados hombre. Número de empleados } \\
\text { mujer }\end{array}$ & & N/A & & \\
\hline & & Total de empleados & Número de empleados total & & $\mathrm{N} / \mathrm{A}$ & & \\
\hline & & Promedio de edad & Suma de edades de los empleados/Total de empleados & & N/A & & \\
\hline & & Empleados por área & Número de empleados en cada área de la empresa & & $\mathrm{N} / \mathrm{A}$ & & \\
\hline & & $\begin{array}{l}\% \text { empleados con } \\
\text { especialización }\end{array}$ & $\begin{array}{l}\text { (Número de empleados con especialización/Total de } \\
\text { empleados)*100 }\end{array}$ & & N/A & & \\
\hline & & $\%$ empleados profesionales & $\begin{array}{l}\text { (Número de empleados con título profesional/Total de } \\
\text { empleados)*100 }\end{array}$ & & N/A & & \\
\hline & & \% empleados tecnólogos & $\begin{array}{l}\text { (Número de empleados con título tecnólogo/Total de } \\
\text { empleados)*100 }\end{array}$ & & $\mathrm{N} / \mathrm{A}$ & & \\
\hline & & $\%$ empleados técnicos & $\begin{array}{l}\text { (Número de empleados con título técnico/Total de } \\
\text { empleados)*100 }\end{array}$ & & N/A & & \\
\hline & & $\%$ empleados bachilleres & $\begin{array}{l}\text { (Número de empleados con título bachiller/Total de } \\
\text { empleados)*100 }\end{array}$ & & N/A & & \\
\hline & & $\begin{array}{l}\text { Antigüedad de los } \\
\text { empleados }\end{array}$ & Suma de años de vinculación/Total de empleados & & N/A & & \\
\hline
\end{tabular}

Revista ENIAC Pesquisa, Guarulhos (SP), V.6, n.2, jun.- dez. 2017 


\begin{tabular}{|c|c|c|c|c|c|c|}
\hline & 2. Formación. & & & & & \\
\hline & $\begin{array}{l}\text { Inversión en formación por } \\
\text { empleado }\end{array}$ & Inversión total en formación/Total de empleados & & N/A & & \\
\hline & Formaciones exitosas & Numero de formaciones realizadas en el año & & $\mathrm{N} / \mathrm{A}$ & & \\
\hline \multirow{12}{*}{ 足 } & \multicolumn{2}{|l|}{ 3. Rotación del personal. } & $\mathbf{0 , 2 8}$ & $\mathbf{0 , 3 0}$ & $\mathbf{0 , 0 3}$ & $92 \%$ \\
\hline & Nuevos empleados & Número de empleados nuevos/total de empleados & 0,00 & 0,10 & & \\
\hline & Retiros no forzados & Número de empleados retirados/Total de empleados & 0,09 & 0,09 & & \\
\hline & Retiros forzados & Número de empleados despedidos/Total de empleados & 0,01 & 0,01 & & \\
\hline & Tasa de reposición & Número de ingresos/número de salidas & 1,00 & 1,00 & & \\
\hline & \multicolumn{2}{|l|}{ 4. Compromiso y motivación. } & $\mathbf{0 , 5 2}$ & $\mathbf{0 , 5 2}$ & $\mathbf{0 , 0 0}$ & $100 \%$ \\
\hline & Promoción de empleados & Número de empleados ascendidos/Total de empleados & 0,05 & 0,05 & & \\
\hline & Reconocimientos & $\begin{array}{l}\text { Número de empleados que sienten reconocidos/Total } \\
\text { de empleados }\end{array}$ & 1,00 & 1,00 & & \\
\hline & Empleados jubilados & Número de empleados jubilados / Total de empleados & 0,03 & 0,03 & & \\
\hline & Sentido de importancia & $\begin{array}{l}\text { Número de empleados que sienten que son tenidos en } \\
\text { cuenta/Total de empleados }\end{array}$ & 1,00 & 1,00 & & \\
\hline & \multicolumn{2}{|l|}{ 5. Resultados. } & 1,00 & 1,00 & $\mathbf{0 , 0 0}$ & $100 \%$ \\
\hline & Satisfacción & $\begin{array}{l}\text { Número de empleados que se sienten } \\
\text { satisfechos/Total de empleados }\end{array}$ & 1,00 & 1,00 & & \\
\hline & \multicolumn{2}{|c|}{ Total } & 1,80 & 1,82 & $\mathbf{0 , 0 3}$ & $99 \%$ \\
\hline
\end{tabular}

Tabla 3: Tablero de control para medición del capital humanoFuente: Los Autores, 2017.

Revista ENIAC Pesquisa, Guarulhos (SP), V.6, n.2, jun.- dez. 2017 


\section{CONCLUSIONES}

El capital humano como elemento fundamental en una organización y por ende en el desarrollo de un encargo, porque a partir del mismo se generan, obtienen y desarrollan los demás capitales; es considerado subjetivo, y su valoración comprende en gran parte a métodos que utilizan información cualitativa.

La investigación evidencia que existen una serie de indicadores que permiten obtener la valoración del capital humano en el encargo, y a partir del resultado de estos indicadores se puede tener conocimiento del estado del capital humano y establecer si es el adecuado para llevar a cabo el trabajo como auditor o por el contrario se debe fortalecer, con los aspectos de detalle que establece cada indicado.

Se visualiza de manera positiva que se siga estudiando este tema en futuras investigaciones, ya que el capital humano es un eje dinamizador muy importante que con una adecuada administración y control, logra resultados favorables en el proceso de planeación, ejecución e informe

\section{Referencias Bibliográficas}

Alama Salazar, Navas López, Martin de Castro (2008). Capital intelectual y resultados empresariales en las empresas de servicios profesionales de España (Tesis doctoral). Universidad complutense de Madrid, Madrid, España.

Gonzales, A., Medina, A. J., y Pérez, E. (2007). El concepto de capital intelectual y sus dimensiones. Investigaciones Europeas de dirección y economía de las empresas, 13(2), 97111.

López, M. A. y Grandino, A. (2005). Capital humano como fuente de ventajas competitivas. Barcelona, España: Netbiblo

Martos, M. S., Jardon, C. M. y Florián, P. (2008). Evaluación y relaciones entre las dimensiones del capital intelectual: El caso de la cadena de la madera de Oberá (Argentina). Intangible capital, 4(2), 67-101.

Hernández, S. F. E. (2006). Conocimiento organizacional: la gestión de los recursos y el capital humano. Cuba: Scielo Cuba.

Contreras, R. D. (2012). Desarrollo del capital humano en las organizaciones. Viveros de la loma: Red tercer mileno

Congreso de la republica Decreto 2420. (2015). Por medio del cual se expide el Decreto Único Reglamentario de las Normas de Contabilidad, de Información Financiera y de Aseguramiento de la Información y se dictan otras disposiciones Bogotá: Diario oficial 49726.

Revista ENIAC Pesquisa, Guarulhos (SP), V.6, n.2, jun.- dez. 2017 